\title{
The Composition of Cosmic Rays at the Knee ${ }^{1}$
}

\author{
Jörg R. Hörandel \\ Department of Astrophysics/IMAPP, Radboud University Nijmegen, \\ P.O. Box 9010, 6500 GL Nijmegen, The Netherlands - http://particle.astro.ru.nl
}

\begin{abstract}
Recent results on the composition of cosmic rays in the energy region from about $10^{14}$ to $10^{18} \mathrm{eV}$ are reviewed.
\end{abstract}

Keywords: cosmic rays, composition, energy spectrum, knee, air showers

PACS: $96.50 . S-$, 96.50.sb, 96.50.sd, 98.70.Sa

\section{INTRODUCTION}

The all-particle energy spectrum of cosmic rays (ionized atomic nuclei from outer space) follows a broken power law $d N / d E \propto E^{\gamma}$. At an energy around $4 \cdot 10^{15} \mathrm{eV}$ the spectral index changes from $\gamma \approx-2.7$ at low energies to $\gamma \approx-3.1$. This structure in the energy spectrum is referred to as "the knee". Understanding the physics behind this "kink" in the energy spectrum is thought to be crucial to understand the origin of Galactic cosmic rays [1].

The origin of cosmic rays is threefold: particles with energies below about $100 \mathrm{MeV}$ are accelerated during solar bursts in magnetic reconnections at the surface of the Sun. Nuclei with energies up to several $10^{17} \mathrm{eV}$ are usually considered to originate within our Milky Way, most likely being accelerated in Supernova Remnants. At the highest energies $\left(>10^{18} \mathrm{eV}\right)$, particles are usually considered to be of extragalactic origin. In the following, we will focus on the composition and origin of Galactic cosmic rays, i.e. cosmic rays with energies between $\approx 1$ and $10^{9} \mathrm{GeV}$.

At energies around $1 \mathrm{GeV}$ individual elements have been identified in direct measurements of cosmic rays above the atmosphere, all elements from the periodic table of elements are present in cosmic rays, e.g. [2]. Their abundance follows roughly the abundance in the local Galactic environment/solar system. Energy spectra for the major cosmic-ray elements from hydrogen to iron have been directly measured up to energies approaching $10^{14} \mathrm{eV}$ (e.g. Fig. 26.1 in Ref. [3]). They follow power laws with a spectral index close to $\gamma \approx-2.7$. Below about $10 \mathrm{GeV}$ the spectra are influenced by solar modulation, i.e. deflection/attenuation in the Heliosphere.

The cosmic-ray flux is strongly falling as a function of energy. Thus, at energies exceeding $10^{14} \mathrm{eV}$ large areas are needed to collect a statistically significant data sample in a reasonable time. The large areas needed, exceeding the size of a soccer field, can only be realized on ground, i.e. below the atmosphere of the Earth. Thus, secondary particles are measured which are produced in extensive air showers, initiated by high-

\footnotetext{
${ }^{1}$ Invited talk, given at the Centenary Symposium 2012: Discovery of Cosmic Rays, June 2012, Denver.
} 

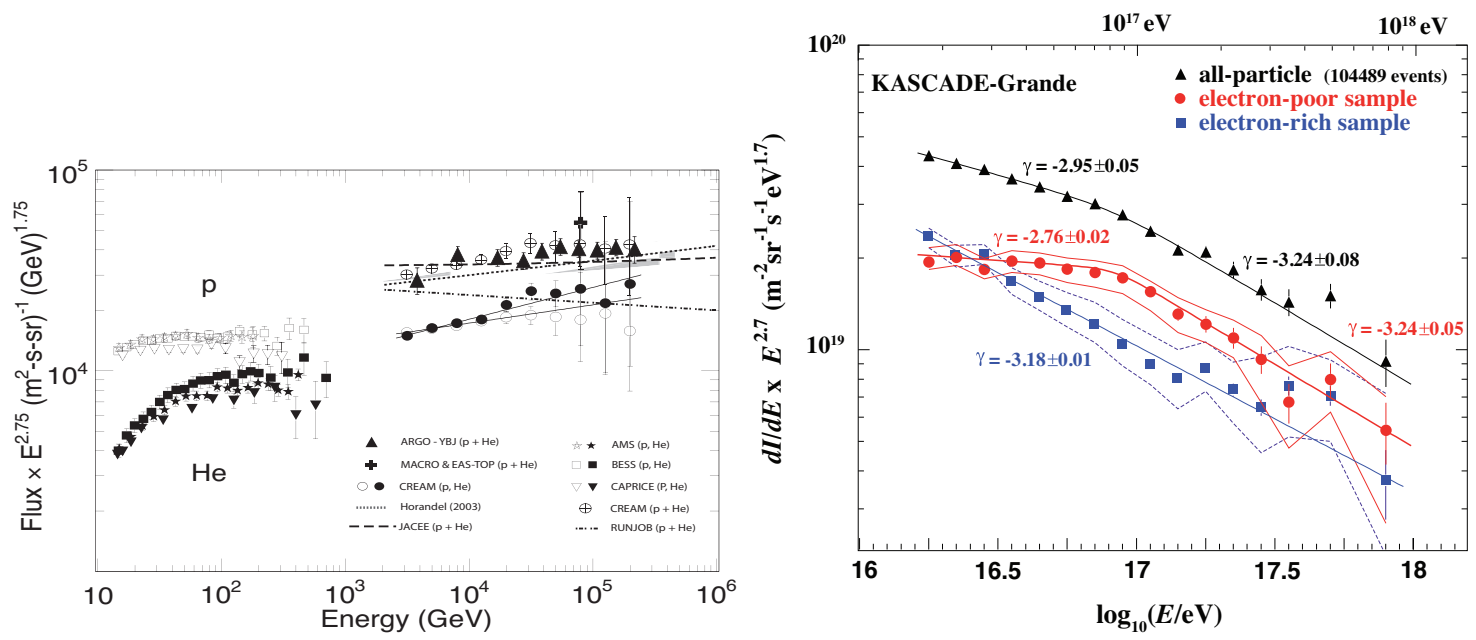

FIGURE 1. Left: Energy spectrum of the "light" cosmic-ray component ( $\mathrm{p}+\mathrm{He})$, as measured by ARGO-YBJ [7]. Right: Energy spectrum of cosmic rays as determined with KASCADE-Grande: allparticle spectrum (black) and heavy component (red) [8].

energy cosmic rays impinging onto the atmosphere.

The intrinsic fluctuations during the development of a particle cascade limit the mass resolution of experiments registering air showers. Typical mass-sensitive observables in such experiments are the depth of the shower maximum in the atmosphere $X_{\max }$ and the ratio of electrons to muons at ground level $N_{e} / N_{\mu}$. Using a Heitler type model for hadronic showers [4] it can be shown that these observables depend on the nuclear mass number of the primary particle $A$ as $X_{\max } \propto-X_{0} \ln A$ and $\lg \left(N_{e} / N_{\mu}\right) \propto-0.065 \ln A$, with the radiation length in air $X_{0}[5,6]$. Thus, if one requires e.g. a resolution of $\Delta \ln A \approx 1$, the experimental accuracies have to be better than $\Delta X_{\max } \approx 36 \mathrm{~g} / \mathrm{cm}^{2}$ or $\Delta\left(N_{e} / N_{\mu}\right) \approx 16 \%$. These values are close to the experimental uncertainties of current air shower experiments. In state-of-the-art air-shower experiments a resolution of the order of $\Delta \ln A \approx 0.8$ has been achieved, i.e. the major cosmic-ray elements from hydrogen to iron can be divided in up to 5 elemental groups (instead of 26 individual elements as registered in direct measurements above the atmosphere).

\section{RECENT RESULTS}

$A R G O-Y B J$. The ARGO-YBJ experiment is situated at the Yangbajing International Cosmic-Ray Observatory, located at an altitude of about $4300 \mathrm{~m}$ a.s.l. in Tibet [7]. The detector consists of an air-shower array, made of a full-coverage RPC carpet. The energy spectrum of the light cosmic-ray component (protons and helium) has been measured in the energy range from 1 to $300 \mathrm{TeV}$, as depicted in Fig. 1 (left). Results of the CREAM balloon experiment are in good agreement with the air-shower data from ARGO. The latter are also compatible with a parameterization of the world data [9], as can be inferred from the figure. This agreement in the energy region of overlap between direct and indirect cosmic-ray measurements is a valuable test of the simulation codes used to 

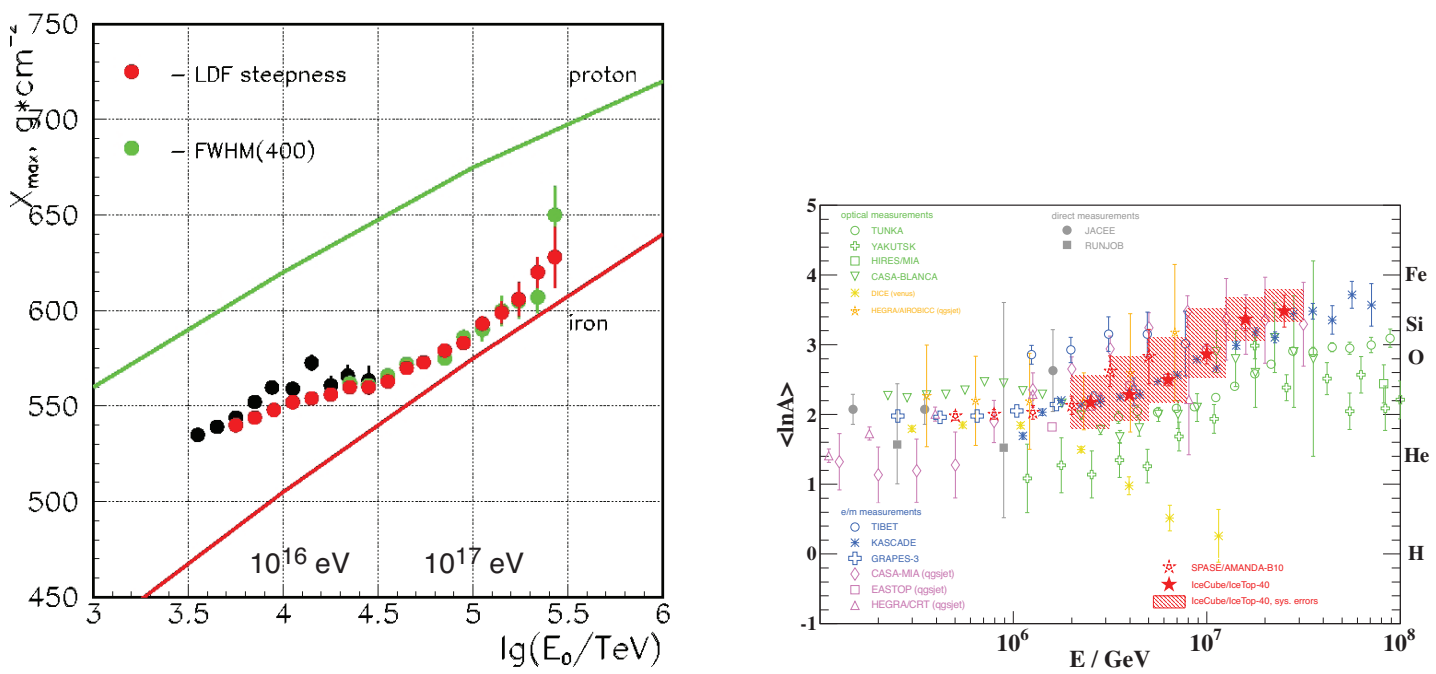

FIGURE 2. Left: Average depth of the shower maximum as a function of energy, measured by Tunka [16]. Right: Mean logarithmic mass as a function of energy obtained by IceCube/IceTop [17].

interpret the air shower data.

KASCADE(-Grande). The KASCADE experiment, located in Germany measured simultaneously all three air shower components [10]. The number of electrons and muons in air showers has been used to unfold the energy spectra for five groups of elements $[11,12]$. The results indicate that the knee is caused by the fall-off of the energy spectrum of protons, followed by subsequent cutoffs of heavier elements, approximately proportional to the rigidity $(E / Z)$ of the individual elements. This observation has been confirmed by independent measurements, using a muon tracking detector to determine the average production depth of muons in the atmosphere [13].

The experiment has been extended to cover an area of about $0.5 \mathrm{~km}^{2}-$ KASCADEGrande [14]. The all-particle energy spectrum has been measured in the energy range from $10^{16}$ to $10^{18} \mathrm{eV}$, derived from measurements of the total number of charged particles and the total muon number of muons [15]. The energy spectrum exhibits strong hints for a hardening of the spectrum at approximately $2 \cdot 10^{16} \mathrm{eV}$ and a significant steepening at $8 \cdot 10^{16} \mathrm{eV}$. The data have also been used to divide the primary cosmic rays in a light and a heavy group and to determine the respective energy spectra, as shown in Fig. 1 (right) [8]. One recognizes a fall-off of the heavy component at an energy around $8 \cdot 10^{16} \mathrm{eV}$. Such a behavior is expected, assuming a rigidity dependent cut-off of the individual cosmic-ray elements.

Based one these measurements, one expects that the average composition becomes heavier as a function of energy up to about $10^{17} \mathrm{eV}$ and again lighter above. Such a behavior is confirmed by the following two observations.

Tunka. The Tunka-133 air shower array with about $1 \mathrm{~km}^{2}$ geometric acceptance area is installed in the Tunka Valley $(50 \mathrm{~km}$ from Lake Baikal) [16]. It measures the 


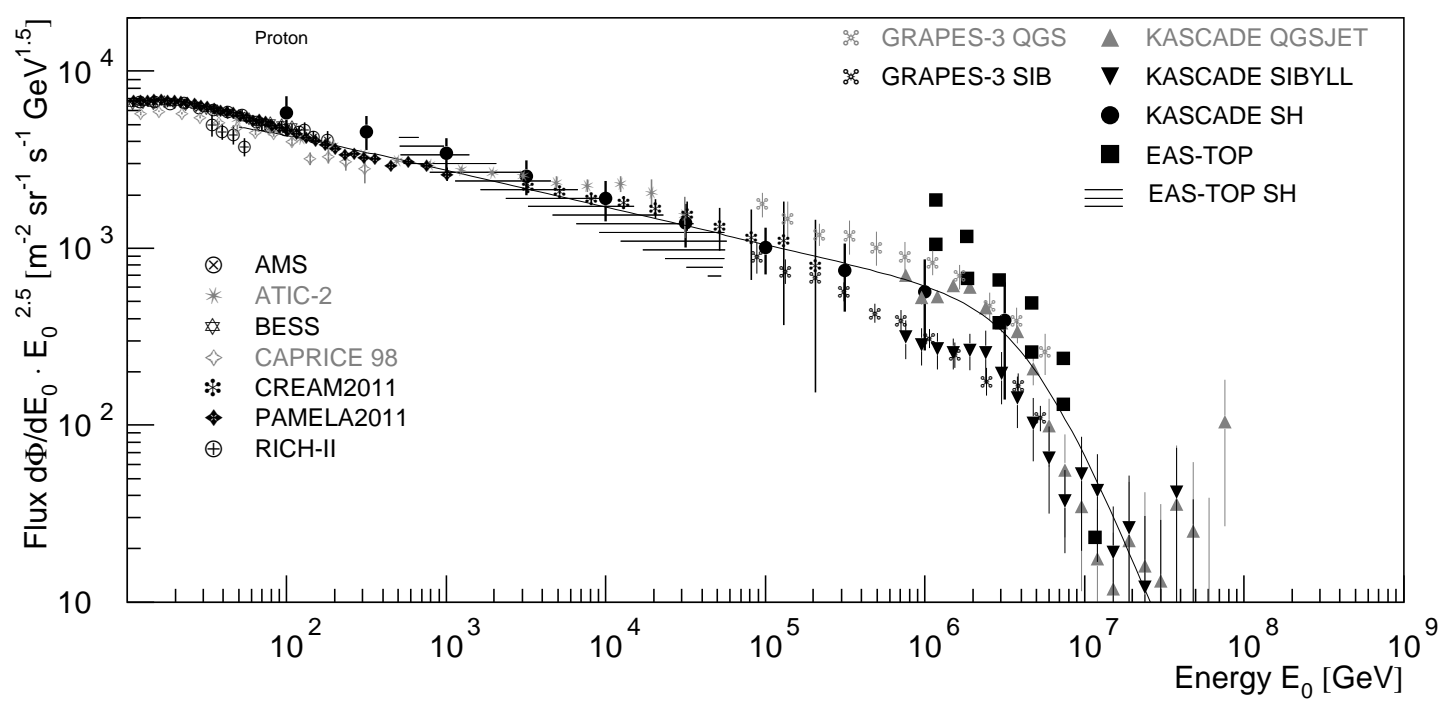

FIGURE 3. Energy spectrum of protons in Galactic cosmic rays as measured by the direct experiments AMS [18], ATIC-2 [19], BESS [20], CAPRICE 98 [21], CREAM2011 [22], PAMELA2011 [23], and RICH-II [24] and the air shower experiments GRAPES [25], EAS-TOP (electrons and muons) [26] (unaccompanied hadrons) [27], and KASCADE (electrons and muons) [11] (unaccompanied hadrons) [28]. Two hadronic interaction models have been used to interpret the data from the GRAPES and KASCADE experiments. The line represents a parameterization according to the Poly Gonato model [9].

Čerenkov component of air showers with an array of 133 open Čerenkov detectors. The average depth of the shower maximum as measured by Tunka is presented in Fig. 2 $(l e f t)$. The lines represent predictions for primary protons and iron nuclei. It can be recognized that as a function of energy, the average cosmic-ray composition becomes heavier (approaching iron) up to about $10^{17} \mathrm{eV}$ and again lighter at higher energies.

IceCube/IceTop. The IceCube neutrino telescope at the South Pole is also a large $\mathrm{km}^{2}$-scale detector for muons from extensive air showers, complemented by an array of detectors on the surface (IceTop) to register the charged particles in air showers [17]. First results on the mass composition of cosmic rays have been obtained from data taken already during the construction of the detector. The mean logarithmic mass derived from one month of data with about half the detector is depicted in Fig. 2 (right). The measurements clearly indicate a rising mean mass as a function of energy. Results up to energies exceeding $10^{17} \mathrm{eV}$ are expected soon with the full detector being operational since 2010 and it will be interesting to see, if a trend to a lighter composition, as discussed above, will be found as well by IceCube at energies exceeding $10^{17} \mathrm{eV}$.

\section{THE COMPOSITION OF GALACTIC COSMIC RAYS}

A compilation of world data from direct and indirect measurements of cosmic rays for four elemental groups is given in Fig. 3 (protons), Fig. 4 (helium nuclei), Fig. 5 (CNO- 


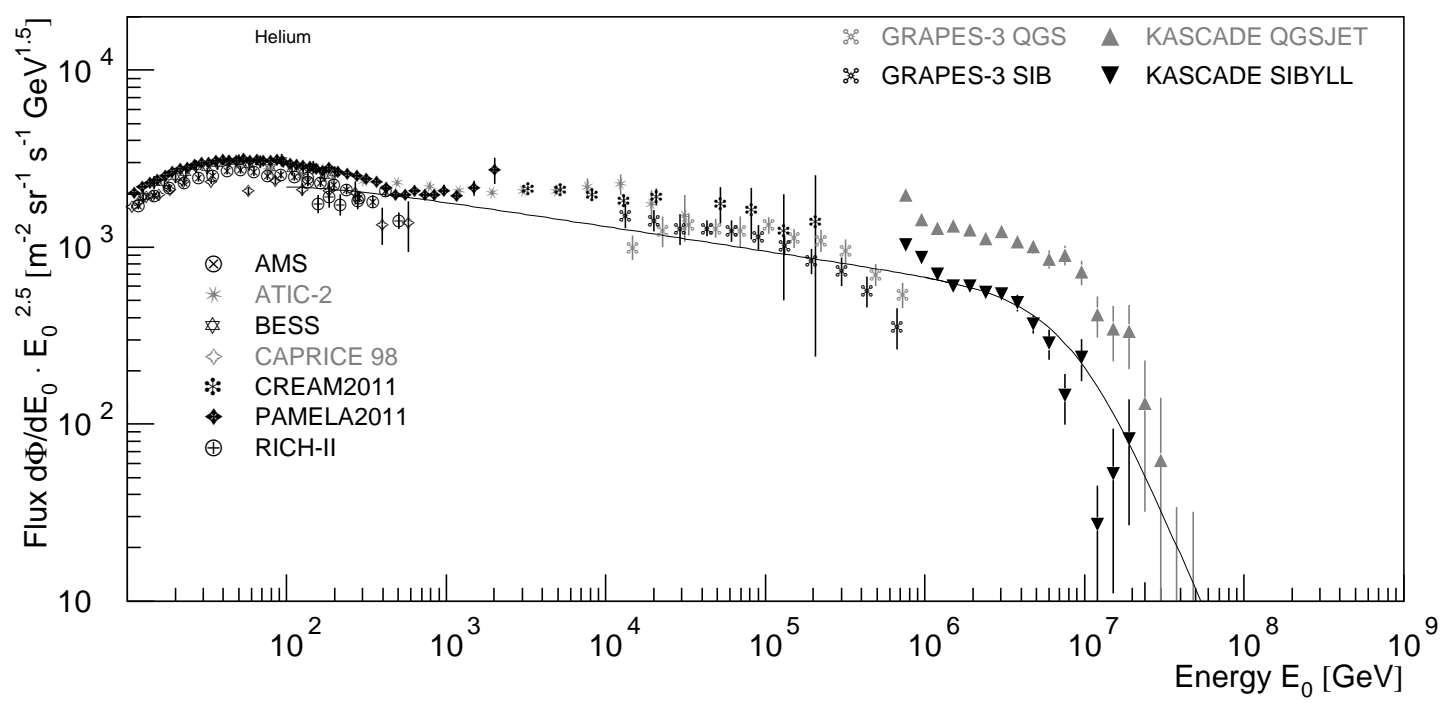

FIGURE 4. Energy spectrum of helium nuclei in Galactic cosmic rays as measured by the direct experiments AMS [18], ATIC-2 [19], BESS [20], CAPRICE 98 [21], CREAM2011 [22], PAMELA2011 [23], and RICH-II [24] and the air shower experiments GRAPES [25], and KASCADE [11]. Two hadronic interaction models have been used to interpret the data from the GRAPES and KASCADE experiments. The line represents a parameterization according to the Poly Gonato model [9].

group nuclei), and Fig. 6 (iron-group nuclei). Here we restricted ourself to "modern" measurements. Older data are included in previous compilations [9, 2]. The energy is given as total energy per particle. Direct measurements above the atmosphere (on balloons and space crafts) extend to almost $10^{6} \mathrm{GeV}$ and at higher energies air shower measurements set in.

To guide the eye the lines represent a parameterization according to the Poly Gonato model with a rigidity dependent cut-off and a constant $\Delta \gamma$ (see Ref. [9] for details) with the following parameter range for the nuclear charge number $Z$ : Fig. 3 protons $Z=1$, Fig. 4 helium $Z=2$, Fig. 5 CNO group $Z=5-12$, Fig. 6 iron group $Z=26-92$.

These figures reflect the present status of our understanding of the elemental composition of Galactic cosmic rays. Several common features can be recognized. At low energies, the flux is influenced by magnetic fields in the heliosphere (solar modulation). At higher energies the spectra follow approximately a power law. Finally, at energies exceeding $10^{15} \mathrm{eV}$ the spectra exhibit a fall-off, which is roughly proportional to the charge of the respective nuclei $E_{c} \approx Z \cdot 4 \cdot 10^{15} \mathrm{eV}$.

A closer look reveals some more properties. An often discussed issue is the spectral slope of protons and helium nuclei. As can be inferred from Figs. 3 and 4, the spectrum of helium is slightly flatter $(\gamma=-2.64 \pm 0.02)$ as compared to protons $(\gamma=-2.71 \pm 0.02)$. Looking closely at the proton and helium spectra, a structure might be visible around $200 \mathrm{GeV}$. Above this energy, the spectra follow power laws, which extend into the air-shower energy region, where ultimately a cut-off is observed. Below about $200 \mathrm{GeV}$, both proton and helium exhibit a "bump", before the solar modulation yields to a depression at lowest energies. This feature is sometimes referred to as "spec- 


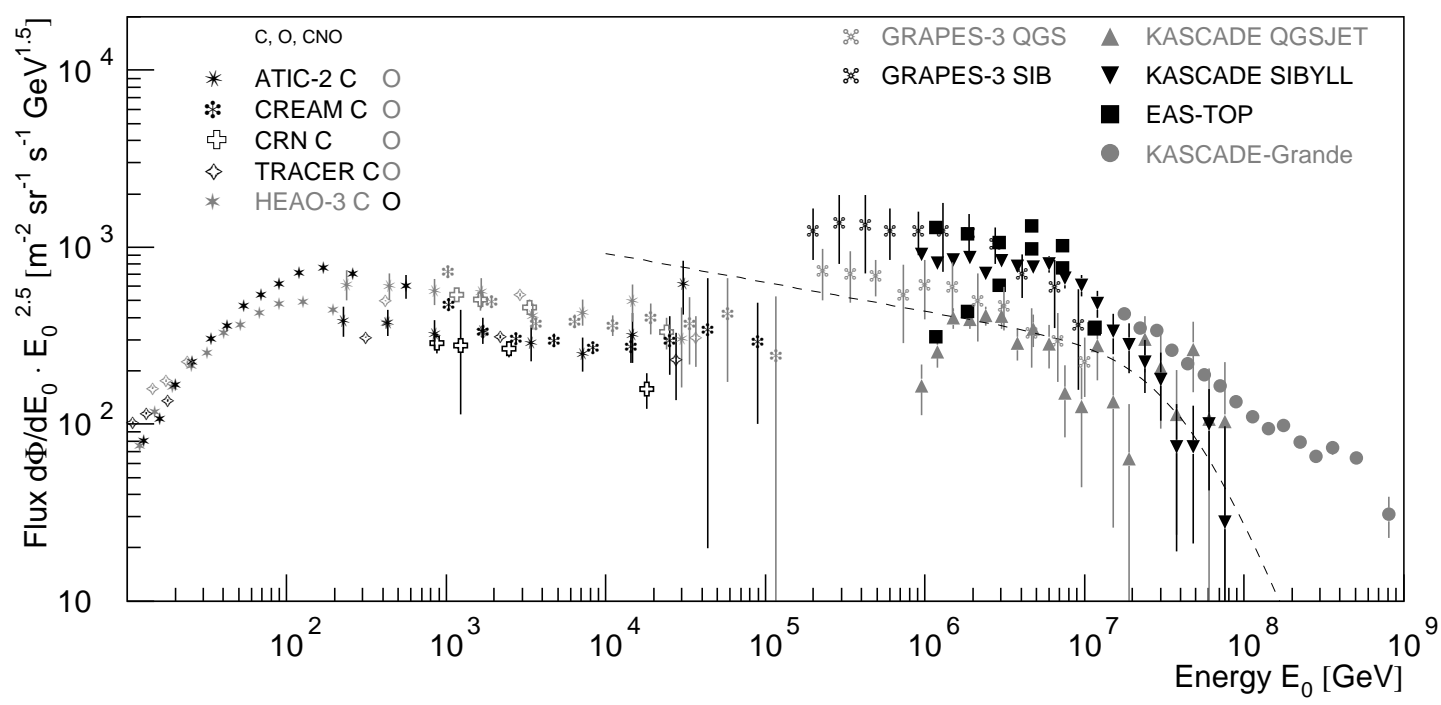

FIGURE 5. Energy spectrum of nuclei from the CNO group in Galactic cosmic rays as measured by the direct experiments ATIC-2 [19], CREAM [22], CRN [29], HEAO-3 [30], TRACER [31] and the air shower experiments GRAPES [25], EAS-TOP [26], KASCADE [11], and KASCADE-Grande (light) [8]. Two hadronic interaction models have been used to interpret the data from the GRAPES and KASCADE experiments. The direct measurements have single-element resolution, i.e. measure the flux of carbon and oxygen nuclei. Air shower experiments can only resolve elemental groups. The line represents a parameterization according to the Poly Gonato model [9].

tral hardening" [22]. However, from Figs. 3 and 4 it appears as there are two cosmic-ray components, one below $200 \mathrm{GeV}$ and a second one at higher energies. It should also be noted that the effect is very subtle and one may ask if systematic effects in the experiments are understood to such a precision, in particular, since the energy corresponds roughly to the transition between two experimental techniques: from magnet spectrometers (at low energies) to calorimeters.

Looking at the CNO and iron groups, it may be noted that the recent KASCADEGrande data (Fig. 1, right) extend previous measurements to higher energies and a cutoff is now also clearly visible in the iron group. Since protons and helium nuclei have already reached their cut-off energies, the "light" component in Fig. 1, right corresponds most likely to the CNO group. An interesting behavior can be observed for the iron group: two hadronic interaction models (QGSJET and SIBYLL) have been used to interpret the air shower data measured by GRAPES and KASCADE. For the interaction model QGSJET a "dip" is visible for both experiments at energies around $10^{6} \mathrm{GeV}$. To derive the spectra the correlations between the number of electrons and muons in the showers are investigated. QGSJET is not compatible with the measured distributions around energies of $10^{6} \mathrm{GeV}$. This yields the depression in the iron spectrum, consistently observed by both experiments. Such a behavior has been observed earlier, for a detailed discussion see also Ref. [11] and [12]. It might also be worth to mention that the recent KASCADE-Grande data for the heavy/iron component are right on top of the predictions of the Poly Gonato model (published a decade before the measurements). 


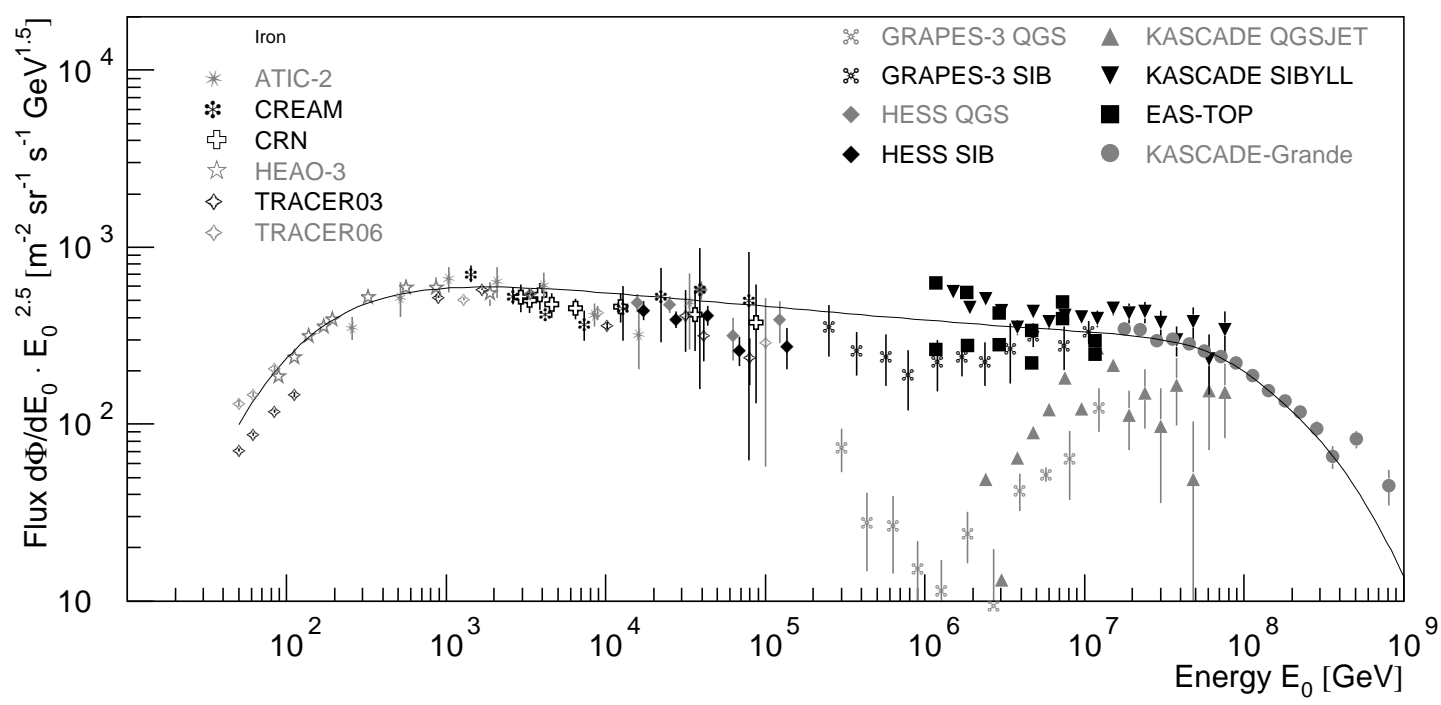

FIGURE 6. Energy spectrum of nuclei from the iron group in Galactic cosmic rays as measured by the direct experiments ATIC-2 [19], CREAM [22], CRN [29], HEAO-3 [30], TRACER 2003 [31] and 2006 [32] and the air shower experiments GRAPES [25], H.E.S.S. direct Čerenkov light [33], EAS-TOP [26], KASCADE [11], and KASCADE-Grande (heavy) [8]. Two hadronic interaction models have been used to interpret the data from the GRAPES and KASCADE experiments. The direct measurements have single-element resolution, i.e. measure the flux of iron nuclei. Air shower experiments can only resolve elemental groups. The line represents a parameterization according to the Poly Gonato model [9].

\section{HADRONIC CROSS SECTIONS AND LHC DATA}

Air showers measured with the Pierre Auger Observatory have been used to derive the proton-air interaction cross section at a center-of-mass energy of $57 \mathrm{TeV}$ [34]. For the proton-air cross section at $(57 \pm 6) \mathrm{TeV}$ a value of

$$
\sigma_{p-a i r}=\left[505 \pm 22(\text { stat })_{-15}^{+20}(\text { syst })\right] \mathrm{mb}
$$

has been derived. Using Glauber theory a value for the inelastic proton-proton cross section has been derived:

$$
\sigma_{p p}=\left[92 \pm 7(\text { stat })_{-11}^{+9}(\text { syst }) \pm 7(\text { Glauber })\right] \mathrm{mb} .
$$

The Auger value is shown together with accelerator measurements, including recent LHC data in Fig. 7. Also shown are predictions from different hadronic interaction models. It should be noted, that the recent Auger results and the recent LHC measurements are at the lower boundary of the range predicted by the various interaction models.

The recent results indicate lower values for the proton-air and proton-proton cross sections. A lower cross section has already been predicted in 2003 [35]. In this work the mean logarithmic mass derived from air shower observations has been systematically investigated. In particular, a discrepancy has been found between experiments observing the depth of the shower maximum and experiments registering the number of secondary particles at ground. It has been shown that a smaller proton-proton and consequently 


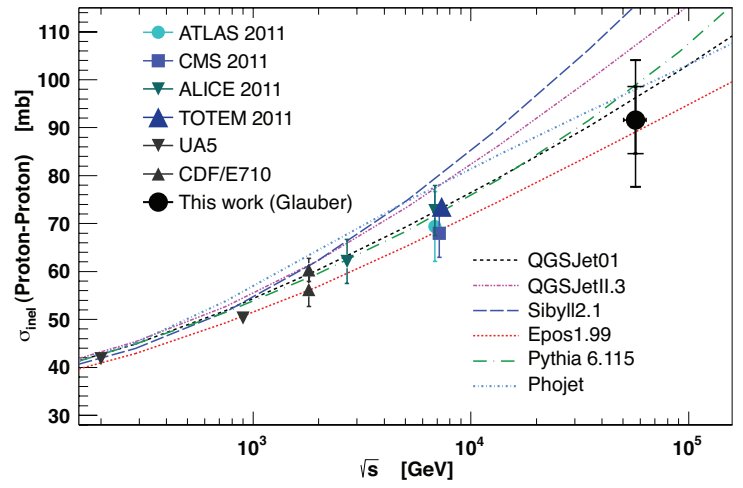

FIGURE 7. Inelastic proton-proton cross section as a function of the center-of-mass energy as measured by various accelerator experiments and recently derived from air shower data by the Pierre Auger collaboration [34]. The lines represent predictions from different hadronic interaction models.

also proton-air cross section reduces the discrepancy in the derived mean logarithmic mass from the different classes of observables. The recent results confirm this earlier findings and this example illustrates how the new LHC measurements directly influence the interpretation of air shower data.

In the past, various ideas have been discussed as possible origin of the knee in the energy spectrum of cosmic rays, among them were ideas about new types of (hadronic) interactions in the atmosphere, see e.g. [36]. The recent LHC results exhibit a fair agreement with predictions of hadronic interaction models using standard physics, see e.g. $[37,38]$. Hence, it can be concluded that the knee in the energy spectrum is not caused by new physics in the atmosphere, it is rather of astrophysical origin.

\section{THE ORIGIN OF GALACTIC COSMIC RAYS}

The origin of the knee in the energy spectrum is often assumed to give hints towards the origin of Galactic cosmic rays. In the literature various scenarios are discussed as possible origin for the knee. They can be grouped in four classes [36]: (i) the finite energy reached during the acceleration process (presumably in Supernova Remnants), (ii) leakage from the Galaxy during the diffusive propagation process, (iii) interaction with background particles during the propagation process, and (iv) new hadronic interactions within the atmosphere, which transport a fraction of the energy into unobserved channels.

The third class can most likely be excluded due to to measurements of the composition of cosmic rays in the energy region around $10^{16}$ to $10^{17} \mathrm{eV}$ [36]. The interactions with background particles would result in a break-up of heavy nuclei, in which many light particles, such as protons and $\alpha$ particles are produced. Thus, on expects a rather light elemental composition in the energy range mentioned. However, recent data indicate a heavy composition in this energy regime, see e.g. Fig. 2.

Also the fourth class can be disregarded due to recent measurements. Air shower data from the KASCADE experiment have been used to check the predictions of hadronic 


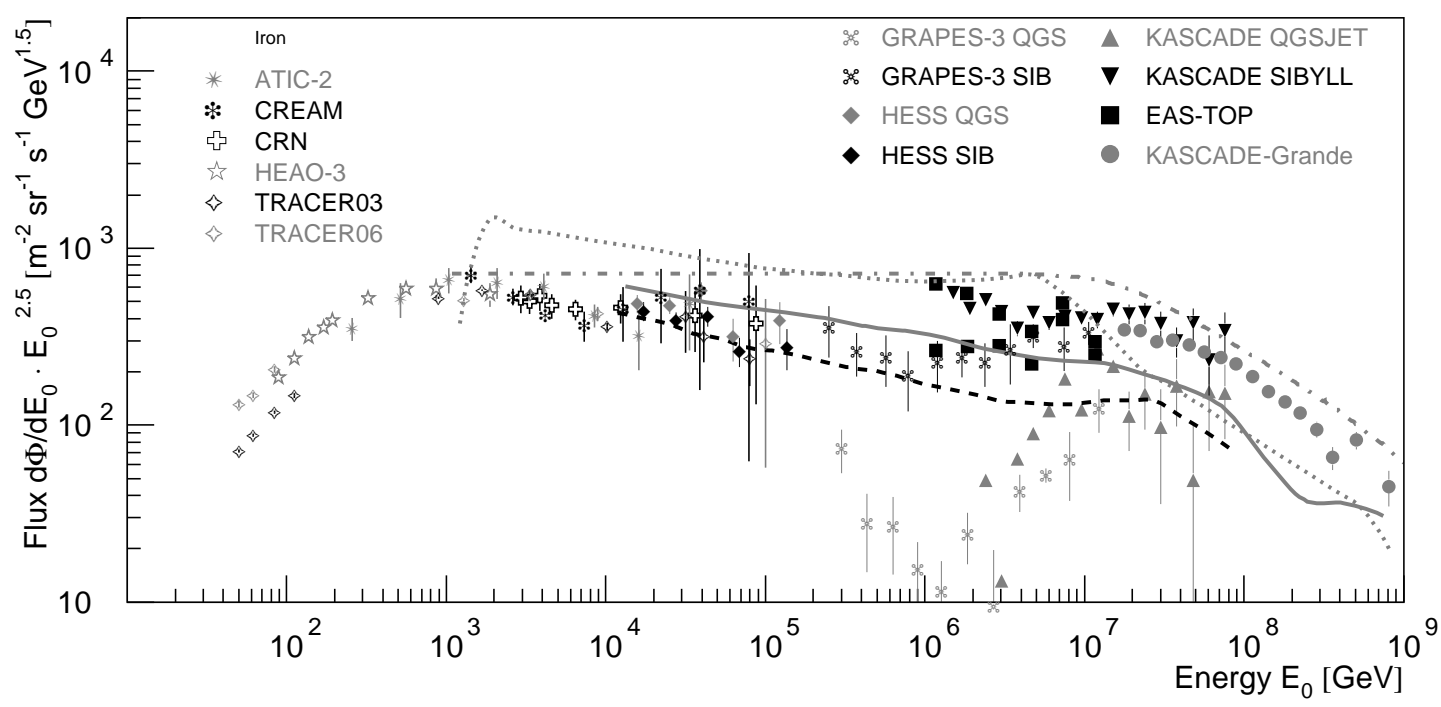

FIGURE 8. Energy spectrum of the cosmic-ray iron group. Experimental data as in Fig. 6. The lines indicate spectra for models explaining the knee due to the maximum energy attained during the acceleration process according to Sveshnikova et al. [39] (-), Berezhko et al. [40] (- - ), Stanev et al. [41] ( . ), and Kobayakawa et al. [42] (---·).

interaction models. A quantitative analysis demonstrates, that the predictions of the models agree with measured observables on the $10 \%$ level [47]. A big step forward in the understanding of hadronic interactions are the results from the Large Hadron Collider (LHC). The design center-of-mass energy of $14 \mathrm{TeV}$ corresponds to a laboratory energy of $10^{17} \mathrm{eV}$, i.e. well above the knee in the energy spectrum. The recent LHC results exhibit a fair agreement with predictions of hadronic interaction models using standard physics, see e.g. $[37,38]$. All this information together leaves very little room for new type of interactions inside the atmosphere. Thus, it can be concluded that the knee in the energy spectrum is not caused by new physics in the atmosphere, it is rather of astrophysical origin.

Predictions of astrophysical models are illustrated in Figs. 8 and 9 for the cosmicray iron group. A comparison of the model predictions with measured data for the light groups is given in Ref. [2]. Models describing the acceleration of cosmic rays in Supernova Remnants predict a maximum energy attained during the acceleration process, which is proportional to the nuclear charge number of the respective atomic nuclei $Z$ and the strength of the magnetic field $B$ in the acceleration region $E_{\max } \propto Z \cdot B$. The predictions of such models are depicted in Fig. 8. A second class of models describes the diffusive propagation in the Galaxy. In this models one finds a maximum rigidity $E / Z$ above which the nuclei cannot be any more effectively magnetically bound to the Galaxy and the particles leak out of the Milky Way. The predictions of such models are shown in Fig. 9. For a more detailed discussion of the models the reader is referred to Ref. [36].

The different models exhibit some scatter, however, the magnitude of the differences is of the same order as the accuracy of the measurements. A general trend can be 


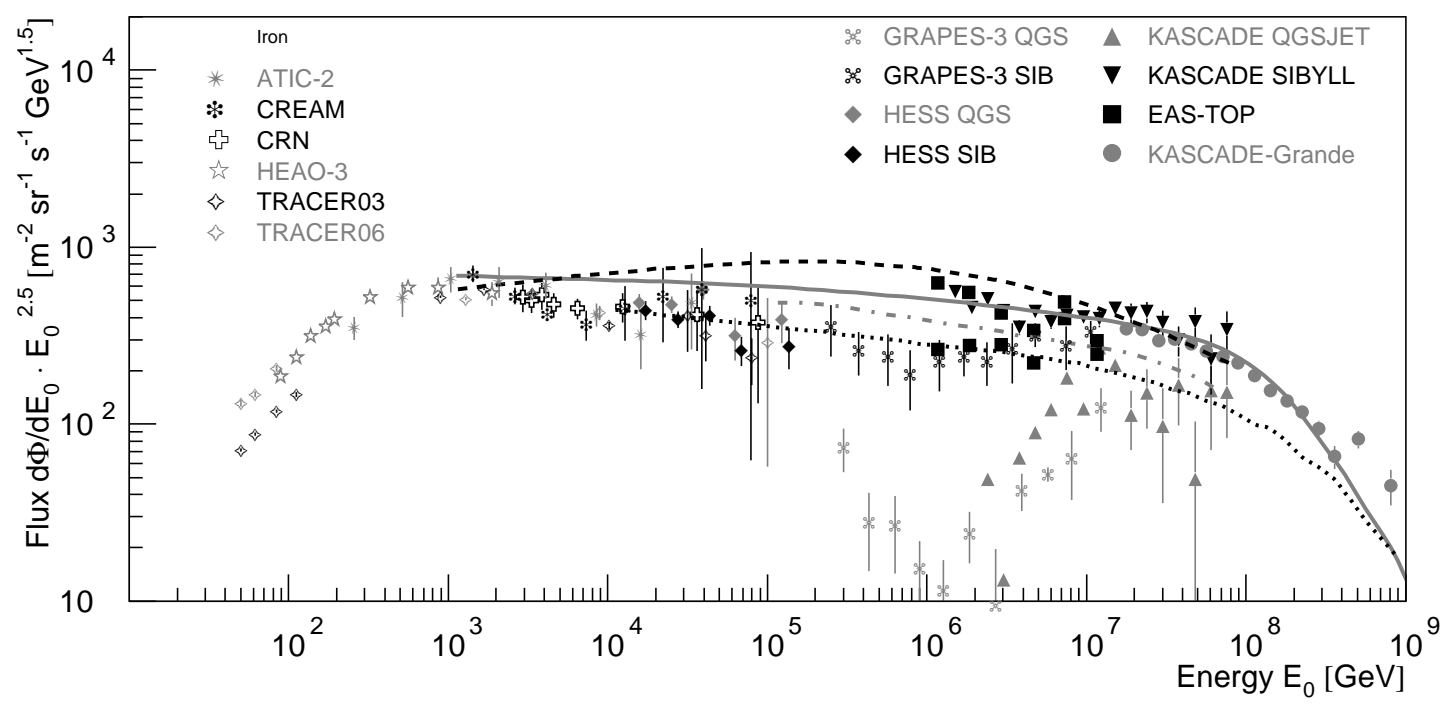

FIGURE 9. Energy spectrum of the cosmic-ray iron group. Experimental data as in Fig. 6. The lines indicate spectra for models explaining the knee as an effect of the propagation process according to Hörandel \& Kalmykov et al. [43] (-), Ogio et al. [44] (---), Roulet et al. [45] (..), as well as Völk et al. [46] (--*).

observed: all models shown describe well the fall-off of the iron group as seen in the measurements. I would like to put some emphasize on the predictions of Ref. [43]. It has been shown that the propagation process alone results in a very soft bend of the individual spectra, not compatible with the measured strong fall-off (see Figs. 3 to 6). In addition, a rigidity dependent maximum energy in the sources had to be included to describe the measured sharpness of the cut-offs. Thus, I would like to conclude that the origin of the knee in the energy spectrum is a combination of the maximum energy attained during the acceleration process and leakage from the Galaxy. This fits very well with the "standard picture" of Galactic cosmic rays, being accelerated in Supernova Remnants and diffusively propagating through the Milky Way.

\section{ACKNOWLEDGMENTS}

The author thanks his colleagues from TRACER, KASCADE-Grande, and the Pierre Auger Observatory for fruitful discussions.

\section{REFERENCES}

1. J. Blümer, R. Engel, and J. Hörandel, Prog. Part. Nucl. Phys. 63, 293 (2009).

2. J. Hörandel, Adv. Space Res. 41, 442 (2008).

3. J. Beringer et al., Phys.Rev. D86, 010001 (2012).

4. J. Matthews, Astropart. Phys. 22, 387 (2005).

5. J. Hörandel, Nucl. Instr. \& Meth. A 588, 181 (2008).

6. J. Hörandel, Mod. Phys. Lett. A 22, 1533 (2007). 
7. S. Mari et al., Proc. 32nd Int. Cosmic Ray Conf., Beijing \#220 (2011).

8. W. Apel et al., Phys.Rev.Lett. 107, 171104 (2011).

9. J. Hörandel, Astropart. Phys. 19, 193 (2003).

10. T. Antoni et al., Nucl. Instr. \& Meth. A 513, 490 (2003).

11. T. Antoni et al., Astropart. Phys. 24, 1 (2005).

12. W. Apel et al., Astropart. Phys. 31, 86 (2009).

13. W. Apel et al., Astropart.Phys. 34, 476 (2011).

14. W. Apel et al., Nucl.Instrum.Meth. A620, 202 (2010).

15. W. Apel et al., Astropart. Phys. 36, 183 (2012).

16. V. Prosin et al., Proc. 32nd Int. Cosmic Ray Conf., Beijing \#184 (2011).

17. A. Tamburro, arXiv:1210.7526 (2012).

18. J. Alcaraz et al., Phys. Lett. B 490, 27 (2000).

19. A. Panov et al., Bull.Russ.Acad.Sci.Phys. 73, 564 (2009).

20. T. Sanuki et al., Astrophys. J. 545, 1135 (2000).

21. M. Boezio et al., Astropart. Phys. 19, 583 (2003).

22. Y. Yoon et al., Astrophys.J. 728, 122 (2011).

23. O. Adriani et al., Science 332, 69 (2011).

24. E. Diehl et al., Astropart. Phys. 18, 487 (2003).

25. Y. Hayashi et al., Proc. 29th Int. Cosmic Ray Conf., Pune 10, 243 (2005).

26. P. Ghia and G. Navarra, Int.J.Mod.Phys. A20, 6817 (2005).

27. M. Aglietta et al., Astropart. Phys. 19, 329 (2003).

28. T. Antoni et al., Astrophys. J. 612, 914 (2004).

29. D. Müller et al., Astrophys. J. 374, 356 (1991).

30. J. Engelmann et al., Astron. \& Astroph. 148, 12 (1985).

31. M. Ave et al., Astrophys. J. 678, 262 (2008).

32. A. Obermeier et al., Astrophys.J. 742, 14 (2011).

33. F. Aharonian et al., Phys. Rev. D75, 042004 (2007).

34. P. Abreu et al., Phys.Rev.Lett. 109, 062002 (2012).

35. J. Hörandel, J. Phys. G: Nucl. Part. Phys. 29, 2439 (2003).

36. J. Hörandel, Astropart. Phys. 21, 241 (2004).

37. T. Pierog et al., Proc. 32nd Int. Cosmic Ray Conf., Beijing \#1169 (2011).

38. J. Hörandel, Proc. 32nd Int. Cosmic Ray Conf., Beijing, Highlight and Rapporteur talks "Rapporteur talk HE2 \& HE3" in press (2012).

39. L. Sveshnikova et al., Astron. \& Astroph. 409, 799 (2003).

40. E. Berezhko and L. Ksenofontov, JETP 89, 391 (1999).

41. T. Stanev et al., Astron. \& Astroph. 274, 902 (1993).

42. K. Kobayakawa et al., Phys. Rev. D 66, 083004 (2002).

43. J. Hörandel et al., Astropart. Phys. 27, 119 (2007).

44. S. Ogio and F. Kakimoto, Proc. 28th Int. Cosmic Ray Conf., Tsukuba 1, 315 (2003).

45. E. Roulet, Int. J. Mod. Phys. A 19, 1133 (2004).

46. H. Völk and V. Zirakashvili, Proc. 28th Int. Cosmic Ray Conf., Tsukuba 4, 2031 (2003).

47. J. Milke et al., Proc. 29th Int. Cosmic Ray Conf., Pune 6, 125 (2005). 